OPEN

SUBJECT AREAS:

PLANT BREEDING

PLANT EVOLUTION

AGRICULTURAL GENETICS

Received

14 September 2014

Accepted

5 December 2014

Published

14 January 2015

Correspondence and requests for materials should be addressed to

S.F. (fukusan@affrc.

go.jp)

* Current address:

Aichi Agricultural

Research Center,

Yazako, Nagakute,

Aichi 480-1 193, Japan

$\S$ Current address:

Aichi Prefectural

Government, Nagoya,

Aichi 460-8501, Japan

† Current address:

Faculty of Life and

Environmental Sciences,

University of Tsukuba,

Tennohdai 1-1-1,

Tsukuba, Ibaraki 305-

8572, Japan

† Current address:

NARO Institute of Crop

Science, Kannondai

2-1-18, Tsukuba, Ibaraki

305-8518, Japan

\section{Gene pyramiding enhances durable blast disease resistance in rice}

\author{
Shuichi Fukuoka' ${ }^{1}$ Norikuni Saka ${ }^{2 *}$, Yuko Mizukami $§$, Hironori Koga ${ }^{3}$, Utako Yamanouchi ${ }^{1}$, \\ Yosuke Yoshioka ${ }^{4} \uparrow$, Nagao Hayashi ${ }^{1}$, Kaworu Ebana' ${ }^{1}$, Ritsuko Mizobuchi' \& Masahiro Yano'
}

\begin{abstract}
${ }^{1}$ National Institute of Agrobiological Sciences, Kannondai 2-1-2, Tsukuba, lbaraki 305-8602, Japan, ${ }^{2}$ Aichi Agricultural Research Center, Mountainous Region Institute, Inahasi, Toyota, Aichi 441-2513, Japan, ${ }^{3}$ Faculty of Bioresources and Environmental Sciences, Ishikawa Prefectural University, Suematsu 1-308, Nonoichi, Ishikawa 921-8836, Japan, ${ }^{4}$ NARO Institute of Vegetable and Tea Science, 360 Kusawa, Ano, Tsu, Mie 514-2392, Japan.
\end{abstract}

Effective control of blast, a devastating fungal disease of rice, would increase and stabilize worldwide food production. Resistance mediated by quantitative trait loci (QTLs), which usually have smaller individual effects than $R$-genes but confer broad-spectrum or non-race-specific resistance, is a promising alternative to less durable race-specific resistance for crop improvement, yet evidence that validates the impact of QTL combinations (pyramids) on the durability of plant disease resistance has been lacking. Here, we developed near-isogenic experimental lines representing all possible combinations of four QTL alleles from a durably resistant cultivar. These lines enabled us to evaluate the QTLs singly and in combination in a homogeneous genetic background. We present evidence that pyramiding QTL alleles, each controlling a different response to $M$. oryzae, confers strong, non-race-specific, environmentally stable resistance to blast disease. Our results suggest that this robust defence system provides durable resistance, thus avoiding an evolutionary "arms race" between a crop and its pathogen.

mproving disease resistance in crops is crucial for stable food production. Although the use of race-specific resistance genes ( $R$-genes) is a major strategy for disease control, these genes are vulnerable to counterevolution of pathogens. New resistance genes are then needed, thus continuing a cycle referred to as an evolutionary "arms race" between crops and pathogens ${ }^{1-3}$. Quantitative trait loci (QTLs), which usually have smaller individual effects than $R$-genes but confer broad-spectrum or non-race-specific resistance, can contribute to durable disease resistance $(\mathrm{DR})^{4,5}$.

DR for blast, a devastating disease of rice (Oryza sativa L.) caused by the fungal pathogen Magnaporthe oryzae, is controlled by multiple genetic loci6-9. Despite the identification of several chromosomal regions for QTLs associated with resistance to $M$. oryzae ${ }^{10}$, most of these QTLs have not been characterized for their spectrum of resistance or for their combined effects. Additionally, previous studies have used materials with heterogeneous genetic backgrounds, in which the presence of variation makes it difficult to assess the effects of the resistance alleles.

One type of DR against $M$. oryzae is found in the durably resistant cultivar Owarihatamochi $(\mathrm{OW})$ and is controlled by four QTLs': pi21, Pi34, qBR4-2, and qBR12-1. pi21 is a loss-of-function mutation of a negative regulator of plant defence ${ }^{11}$, and the candidate genes for Pi34 encode previously uncharacterized proteins ${ }^{12}$. $q B R 4-2$ is a complex genetic locus including three tightly linked loci: $q B R 4-2 a, q B R 4-2 b$, and $q B R 4-2 c^{13}$. The chromosomal regions for $q B R 4-2$ and $q B R 12-1$ have each been located to $R$-gene clusters ${ }^{14}$. Pi63, which is allelic to $q B R 4-2 b$, encodes a nucleotide-binding site-leucine-rich repeat (NBS-LRR) protein whose transcript expression level is associated with the level of resistance ${ }^{15}$. These data suggest that multiple biological mechanisms contribute to this type of DR, and that it would be worthwhile to examine the effects of pyramiding the component QTLs.

In the present study, we separately introgressed the resistance alleles at each of these four loci into a susceptible and well-characterized genotype, Aichiasahi (AA). Then we crossed these lines and their progeny to produce a total of fifteen genotypes containing one to four of the resistance alleles to test all possible gene combinations within a homogeneous genetic background under multiple field environments and in a glasshouse pathogen isolate challenge.

\section{Results}

Resistance to $M$. oryzae in four single-QTL near-isogenic lines. The chromosomal regions contained within four near-isogeneic lines (NILs) for pi21, Pi34, qBR4-2, and $q B R 12-1$ in the genetic background of AA have been 
identified (Supp. Fig. S1). Eight field tests at two locations (Inabu and Tsukuba) revealed that the resistance conferred by each of the four alleles individually was incomplete, had varying magnitude, and was substantially sensitive to environment (coefficient of variation [C.V.] $\geq 15 \%$ ) (Fig. 1a, Table 1). Nevertheless, analysis of variance (ANOVA) revealed that each allele had a significant individual effect $(P<0.001)$ : for pi21, $F(1,827)=1483.031$; for $q B R 4-2$, $F(1,827)=91.495$; for $P i 34, F(1,827)=50.319$, and for $q B R 12-1$, $F(1,827)=13.275$. Similar results were obtained after a glasshouse challenge with eight races of $M$. oryzae that are widely distributed in Japan and a ninth race (IBOS8-1-1) that is aggressively virulent to Pi34 (Supp. Fig. S2). The resistance conferred by $q B R 4-2$ was less effective in field trials with an average temperature above $25^{\circ} \mathrm{C}$ during the 2 weeks before scoring than when temperatures were lower (Table 1). In a glasshouse challenge using fungus race 007.0, the lesion area of the $q B R 4-2$ plants was significantly smaller than that of the recurrent parent (AA) when the plants were incubated at $20^{\circ} \mathrm{C}$ for 1 week before inoculation, but the difference was not significant when the plants were incubated at 24 or $28^{\circ} \mathrm{C}$ (Supp. Fig. S3). Of the four QTLs tested in the glasshouse challenge, only Pi34 significantly reduced the lesion number per leaf when challenged with the seven of eight Japanese races of the fungus, but none of the single QTLs significantly reduced lesion number when challenged with IBOS8-1-1 (Supp. Fig. S2).

Ethylene and salicylic acid (SA) signalling are major components of disease resistance in plants ${ }^{16-18}$. We examined the responses to these signals in the pi21 and Pi34 lines because their resistance was stably detectable in the glasshouse pathogen isolate challenge. Application of an antagonist of ethylene biosynthesis, 2-aminoisobutyric acid (AIB), before inoculation did not alter average lesion area in the plants carrying pi21, whereas AA plants and plants carrying Pi34 had significantly larger lesions than the corresponding untreated controls (Supp. Fig. S4). When $500 \mu \mathrm{M}$ SA was applied before inoculation, plants carrying Pi34 had a significantly smaller average lesion area than untreated plants, whereas the average lesion areas of AA plants and those carrying pi21 were unchanged (Supp. Fig. S4).

Resistance to M. oryzae in QTL pyramid lines. Combinations of resistance alleles reduced lesion area both in field tests (Fig. 1a and b, Table 1) and in glasshouse inoculation tests with the nine previously described races of $M$. oryzae (Fig. 2a). The pi21 resistance allele had the greatest effect on lesion area among the four QTLs when present alone (Supp. Fig. S2), and combination of pi21 with other QTLs cumulatively reduced lesion area against all nine races tested (Fig. 2a). A pyramid of the three QTLs other than pi21 had an average lesion area somewhat higher than that of pi21 alone when tested in the field (Table 1). The line with four resistance alleles (AA$4 \mathrm{RQ}$ ), which carried only $6 \%$ of the donor (OW) genome, had an average lesion area of $\leq 1 \%$, comparable to that of OW. Importantly, the C.V. for lesion area across field tests was smaller in AA-4RQ than in lines with only one or two resistance alleles (Fig. 1a, Fig. 2a, Table 1). In contrast, $R$-gene-mediated resistance fluctuated in field tests, possibly owing to changes in pathogen populations over time (Supp. Table S1). Lesion number per leaf was smaller in AA4RQ than in the line with pi21 alone when the two lines were challenged with the nine races, although the difference was not significant when the lines were challenged with IBOS8-1-1 (Fig. 2b).

Characterization of QTL pyramid lines. In a histological analysis, cells of AA-4RQ infected with a virulent race of $M$. oryzae lacked the hypersensitive response (HR) $)^{19}$ that is often associated with $R$-genemediated resistance, as indicated by the presence of viable cells at infection sites (Supp. Table S2). But cytoplasmic granules, the first

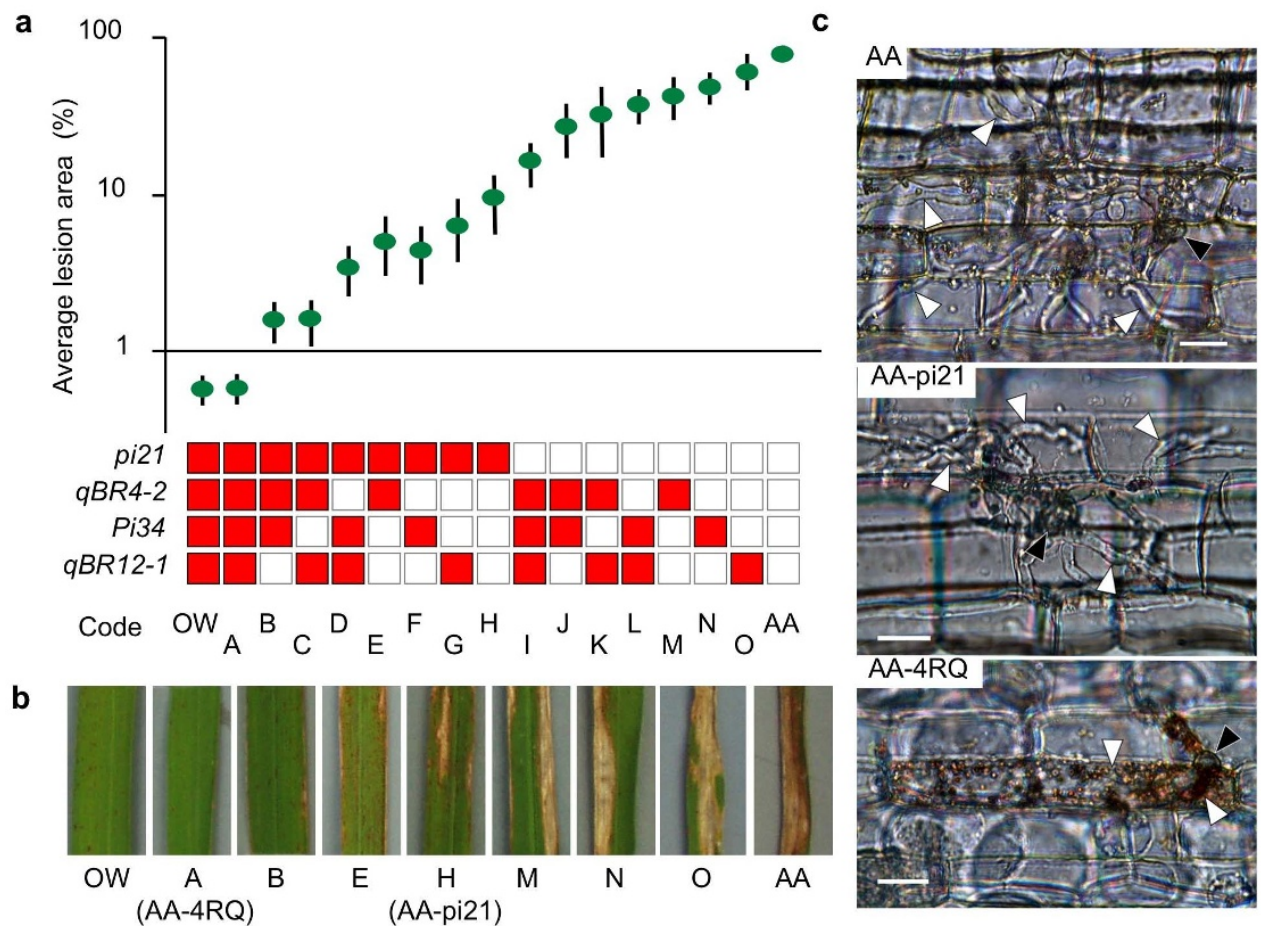

Figure $1 \mid$ Combining alleles of different quantitative trait loci (QTLs) enhances resistance to $M$. oryzae in rice. (a) Average lesion areas of fifteen singleor multiple-QTL lines, the donor parent Owarihatamochi (OW), and the recurrent parent Aichiasahi (AA) across eight field tests. Error bars indicate SD. Genotypes are indicated below the graph. Boxes represent alleles for resistance (red) and susceptibility (white) for each QTL listed at the left. (b) Leaves of 60- to 70-day-old plants from the field evaluation for resistance to M. oryzae. Letters correspond to genotypes shown in (a). (c) Infected cells in leaf sheath epidermal tissue 45 to $48 \mathrm{~h}$ after inoculation. Tissue is from 48-day-old plants infected with M. oryzae race 007.0 (Ina86-137). AA-pi21 and AA-4RQ correspond to genotypes $\mathrm{H}$ and A, respectively, in (a) and (b). The small brown circles in the infected AA-4RQ cell are cytoplasmic granules. Black arrowhead, appressorium; white arrowheads, invading hyphae. Bars represent $20 \mu \mathrm{m}$. 


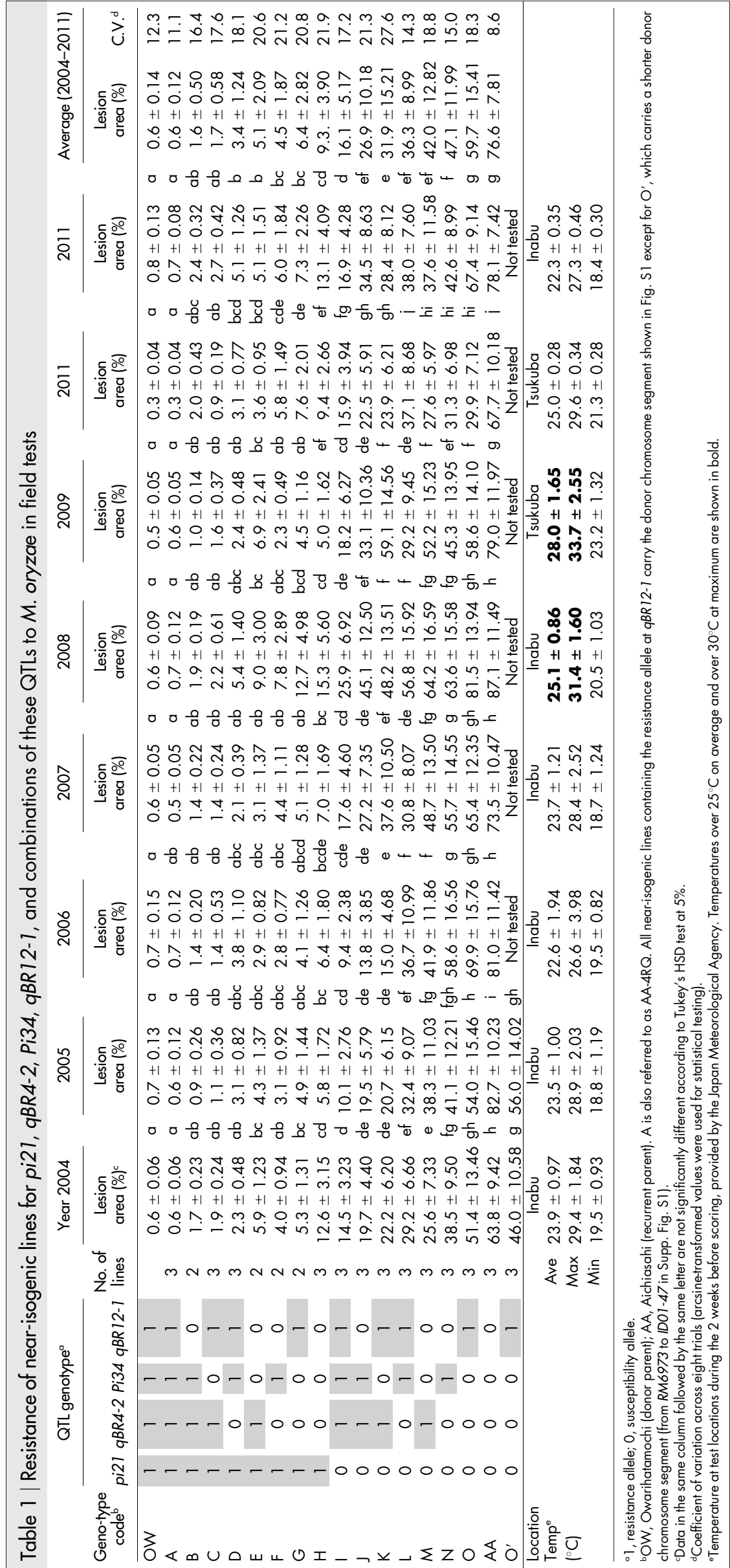




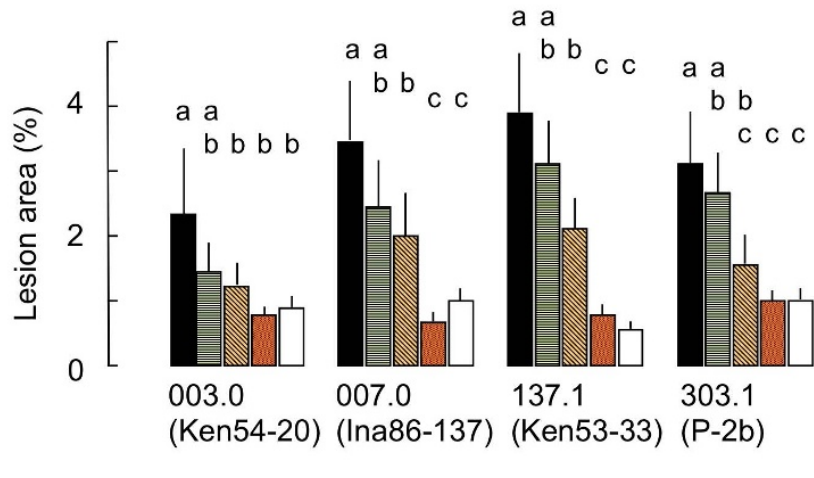

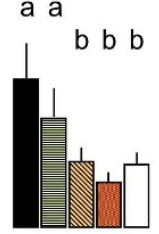

003.0

(Ken54-20)

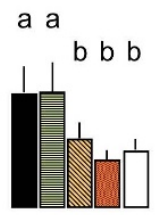

007.0

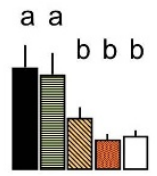

137.1

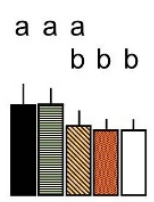

303.1

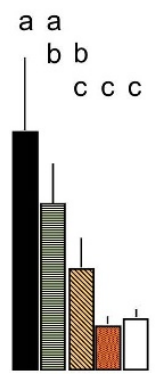

037.3

(Ai79-142)

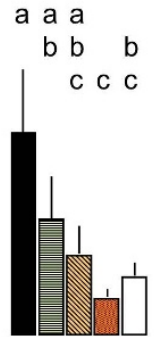

337.3

(Mu-183)

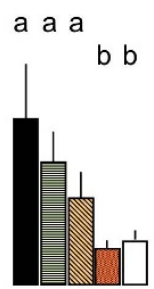

437.1

(Iw81-04) a a a

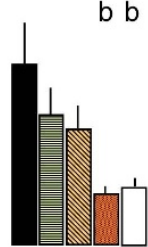

477.1

(Ai74-134) a a

b b

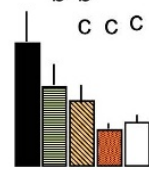

(IBOS8-1-1)

pi21

pi21+qBR4-2

pi21+qBR4-2+Pi34

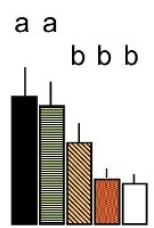

037.3

(Ai79-142)

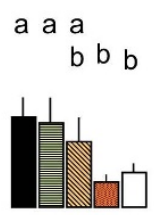

337.3

(Mu-183)

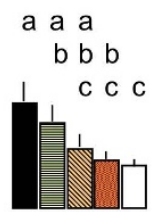

437.1

(Iw81-04) a a a a a

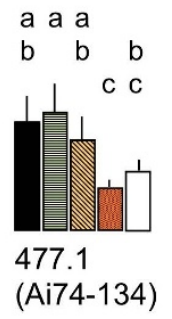
(AA-4RQ)

Figure $2 \mid$ QTL pyramiding enhances resistance to nine blast isolates. (a) Lesion area (\% of total leaf area) and (b) number of lesions per leaf of 35-dayold plants of near-isogenic lines containing pi21 alone or in combination with other QTLs were measured at 7 days after inoculation in a glasshouse inoculation test. A genotype "pi21 + qBR4-2+Pi34+qBR12-1" is also referred to as AA-4RQ. The donor parent, Owarihatamochi, was also included in the test. Isolate IBOS8-1-1 is aggressively virulent to Pi34, and the other eight are differential isolates of M. oryzae widely distributed in Japan. Error bars indicate SD. Values labelled with the same letter do not differ significantly according to Tukey's HSD test at 5\%.

sign of cell death, were observed as early as 45 to $48 \mathrm{~h}$ after inoculation in AA-4RQ plants (Fig. 1c), consistent with the higher expression of pathogenesis-related $(P R)$ genes in AA-4RQ than in Aichiasahi or in plants with pi21 alone (AA-pi21) (Fig. 3). This induction of $P R$ gene expression was not as great as that by $R$ gene-mediated resistance (Fig. 3, Avirulent) at the tested time points. Consequently, the biomass of a virulent race of $M$. oryzae (measured by qPCR targeting the fungal repetitive element Pot2) was dramatically lower in the AA-4RQ plants than in Aichiasahi (Fig. 4).

\section{Discussion}

Cultivars with DR against rice blast have maintained resistance over time, and multiple QTLs lie behind their high levels of resistance ${ }^{6-9}$. Therefore, QTL-mediated resistance is considered to be more durable than $R$-gene-mediated resistance. Cultivars with DR are usually unimproved, and their exotic, often heterogeneous genetic backgrounds make it difficult to predict the potential impact of their DR in the genetic background of elite cultivars. In the present study, we successfully characterized a set of four QTLs in a DR genotype conferring resistance to $M$. oryzae and clearly demonstrated the QTLs' ability to confer resistance under multiple environments and against diverse isolates. The NIL with four resistant QTL alleles showed comparable resistance to the donor, and more stable resistance (i.e., lower C.V.) than lines with only one or two resistance alleles. These observations reinforce the idea that this combination of QTLs with diverse responses against the pathogen underlies DR to M. oryzae in Owarihatamochi.
Previous studies have characterized and cloned three genes for QTL-mediated resistance to $M$. oryzae in rice, namely, pi21, Pi63 and Pi35 $5^{11,15,20}$; these QTLs had the largest effects in the primary genetic mapping studies ${ }^{7,9,21}$. However, QTLs with smaller effects have not yet been characterized owing to the difficulty in controlling experimental noise caused by variation in genetic background and by genotype $\times$ environment interaction. Our study highlighted the importance of minor QTL alleles for improving the durability of resistance by using experimental lines with a highly homogeneous genetic background.

As expected, the resistance conferred by each of the four alleles individually was substantially sensitive to environment. This confirms the need to evaluate QTL effects under multiple environments to identify stable QTL alleles for use in crop improvement. Among the four QTL alleles, only Pi34 significantly reduced lesion number per leaf when present alone, implying that this QTL acts around the time of fungal penetration. Average lesion area is a function of the number of lesions per leaf and the size of each lesion. Resistance conferred by Pi34 is less effective in reducing average lesion area than that conferred by pi21, owing to the ineffectiveness of Pi34 at suppressing lesion development after infection. To elucidate the mechanism(s) of resistance, we examined the response of Pi34 and pi21 plants to altered levels of ethylene and SA, signalling compounds that modulate disease resistance in plants ${ }^{16-18}$. The increased resistance observed in SA-treated Pi34 plants implies that the gene at this QTL is associated with SA signalling. Ethylene induces resistance to a number of diseases when applied before infection, and inhibition 


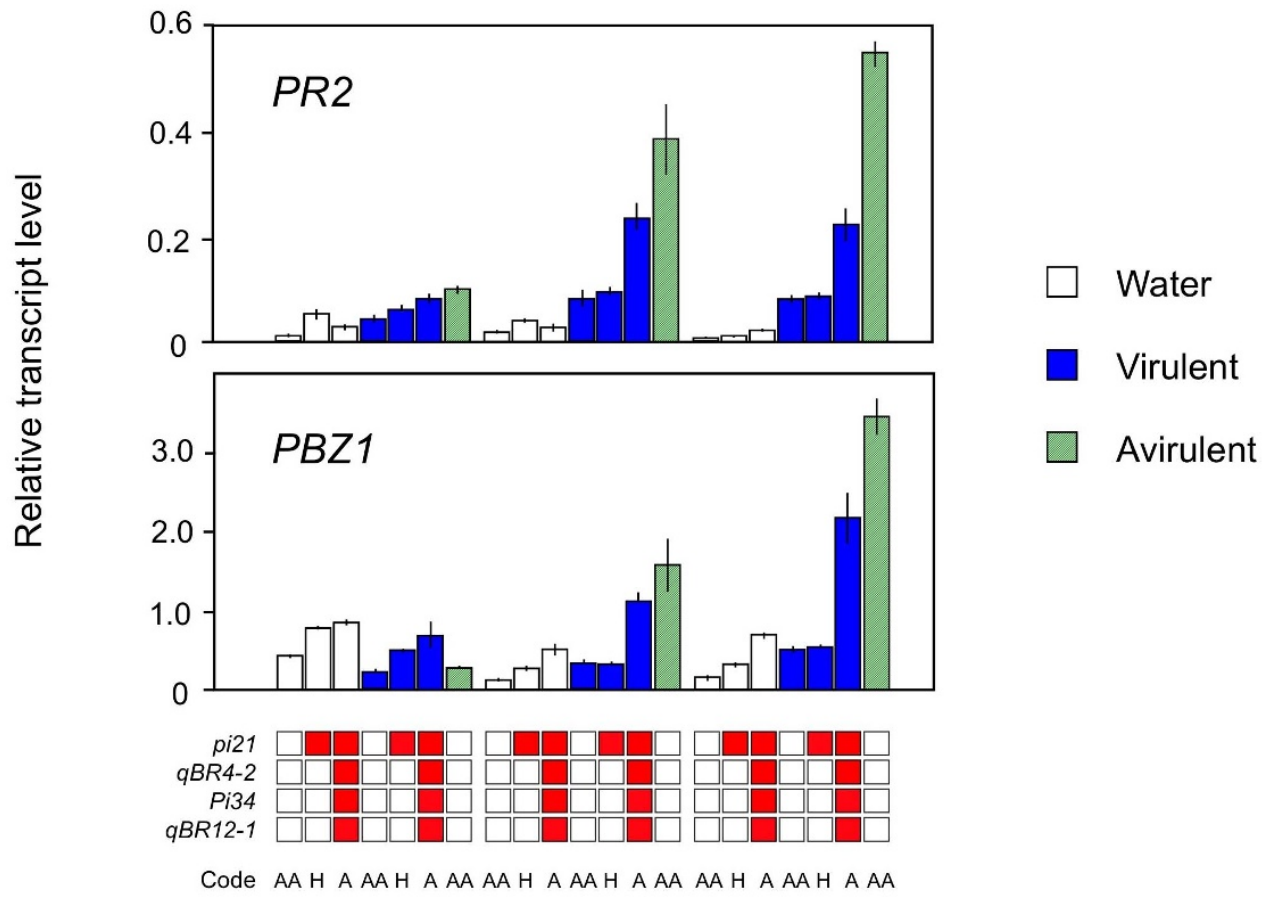

28

32

hr after inoculation

Figure $3 \mid$ A pyramid of four QTLs increases expression of $P R$ genes after inoculation with $M$. oryzae. Graphs show relative expression of $P R$ genes after inoculation with a virulent race (007.0, blue bars), water (open bars), or an avirulent race (001.2, green hatched bars). Genotypes were Aichiasahi, AApi21, and AA-4RQ (AA, H, and A in Fig. 1a and at the bottom of the figure); boxes represent alleles for resistance (red) and susceptibility (white) at pi21, $q B R 4-2, P i 34$, and $q B R 12-1$. Thirty-five-day-old plants were used, and leaves were sampled at 28, 32, and $36 \mathrm{~h}$ after inoculation. Expression of $P R$ genes Pathogenesis-related 2 (PR)2 (Os01t0940700) and Probenazole 1 (PBZ1) (Os12t00555500) was standardized to that of riboubiquitin 2 (Os02t0161900). Error bars indicate SEM $(n=3)$.

of its biosynthesis decreases resistance ${ }^{16}$. Our observations with Aichiasahi and Pi34 plants are consistent with these earlier findings. However, plants containing pi21, a loss-of-function mutant in a proline-rich-protein gene, were insensitive to this treatment, implying that pi21-mediated resistance involves ethylene signalling. We observed temperature dependence of $q B R 4-2$, as reported in other pathosystems ${ }^{22-24}$. Together, these findings confirm that diverse biological functions are involved in QTL-mediated resistance.

Expression analysis and histological study revealed that the fourQTL pyramid line, AA-4RQ, does not induce $\mathrm{HR}$, and that induction of $P R$ genes was not as great as that by $R$-gene-mediated resistance at the tested time points. These observations confirm that the resistance seen in AA-4RQ is of the "susceptible infection type", which shows developing lesions ${ }^{25}$, but it has several characteristics that differ from the typical susceptible infection type. Around 32 or $33 \mathrm{~h}$ after inoculation, the hyphae of a virulent fungus race have just begun moving from the first-invaded host cell to the next cells and the first-invaded host cell is mostly intact, without any sign of defence response ${ }^{26}$. Increased expression of $P R$ genes at this time point in AA-4RQ plants suggests induction of the defence response earlier than in no-QTL or pi21-only plants. This assumption is supported by the lower viability of AA-4RQ plants cells, even without signs of cell death, at $33 \mathrm{~h}$ after inoculation. Thus, AA-4RQ plants allow fungal infection but effectively suppress fungal development in the cell. This strategy may decrease selection pressure and slow the counter-evolution of the pathogen. These observations suggest that this QTL pyramid does not mimic $R$-gene-mediated resistance, but does allow plants to effectively control the disease.

Our results provide evidence that QTL pyramids can confer strong and environmentally stable disease resistance. The rapid breakdown of resistance of cultivars with four $R$-genes ${ }^{27}$ implies that a pyramid of $R$-genes, each posing strong selection pressure against its pathogen, may not improve the durability of resistance. Thus, our findings on QTL pyramids are important for plant breeders because trait stability is a key requirement for sustainable production of agricultural crops. Assembling large numbers of QTL alleles to provide stable agronomic performance is still a time-consuming task owing to a lack of molecular marker information. However, recent progress in genome analysis and breeding methodology will enable a paradigm shift in crop improvement-the construction of ideal genotypes through the use of high-throughput genotyping ${ }^{28,29}$-and this achievement would contribute substantially to global food security.

\section{Methods}

QTLs for resistance to $M$. oryzae in rice. Owarihatamochi (OW) is a cultivar of rice (Oryza sativa) that has maintained resistance to blast disease caused by $M$. oryzae throughout a century of cultivation ${ }^{6}$ and whose resistance is controlled by several QTLs ${ }^{9}$. A major QTL, pi21, explained $45.7 \%$ of the phenotypic variation, and QTLs on chromosomes 4 (qBR4-2) and 12 (qBR12-1) explained $29.4 \%$ and $13.7 \%$, respectively ${ }^{9}$. Pi34, on chromosome 11 , was detected by using a statistical test with a lower probability threshold (PROC GLM program in the Statistical Analysis Systems software, with significance at $P=0.05)^{9}$. The effect of $P i 34$ was validated using Chubu32, a breeding line derived from a DR cultivar ${ }^{12}$.

Development of near-isogenic lines. By performing three to five rounds of backcrossing and marker-assisted selection (MAS), we developed NILs for $q B R 4-2$, $q B R 12-1$, and Pi34, each carrying the resistance allele from $\mathrm{OW}$ in the genetic background of Aichiasahi (AA), a highly susceptible and well-characterized cultivar used in blast resistance studies. A NIL for pi21 in the same genetic background (AApi21) was obtained from a previous study ${ }^{11}$. MAS was conducted to introduce 3- to 24-cM chromosomal fragments containing each QTL from the donor, by using DNA markers described in a previous study': markers C975 and G271 for pi21, C1016 and R738 for $q B R 4-2$, C6 and G257 for Pi34, and OSR20 and G402 for $q B R 12-1$. We used 118 additional DNA markers from the previous study ${ }^{9}$ to confirm that the rest of the genome of each NIL was derived from AA (Supp. Fig. S1). AA carries the R-gene Pia and shows resistance to $M$. oryzae races 001.0, 001.2, and 031.1, but not to the nine races shown in Fig. 2 and Supp. Fig. S2. 


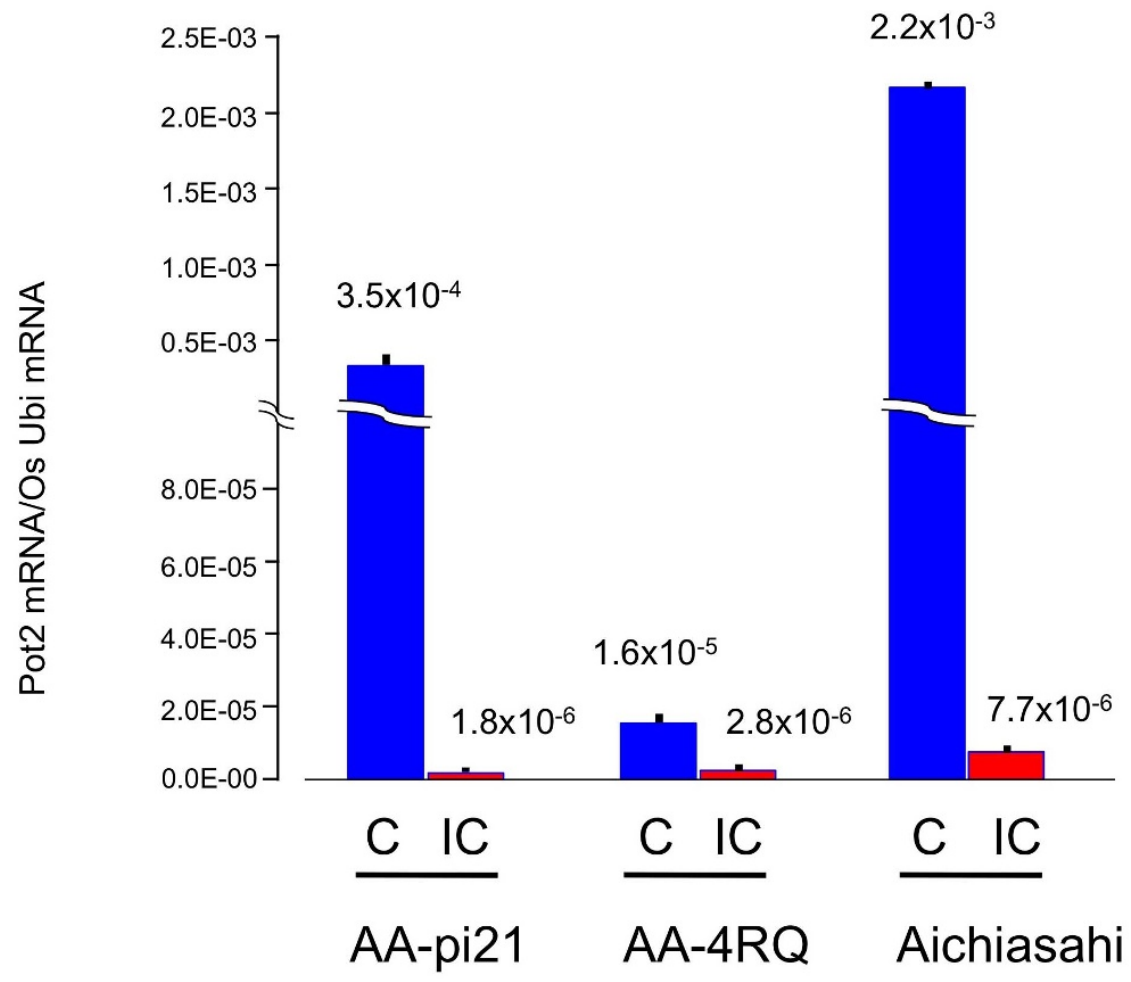

Figure $4 \mid$ A pyramid of four QTLs effectively suppresses growth of $M$. oryzae in inoculated rice leaves. Assessment of fungal growth in leaves of 28 -dayold plants was based on quantitation of the fungal repetitive element Pot 2 by real-time PCR analysis. Each value represents the average and standard deviation of DNA samples from three genotypes (AA-pi21, AA-4RQ, and Aichiasahi) at 4 days after inoculation. Leaf samples were collected from rice seedlings in the glasshouse; each sample contained pooled leaves collected from eight infected seedlings grown in the same tray. C, challenge by virulent race 003.0 (Kyu89-246); IC, challenge by avirulent race 001.0 (93-406). Genotypes AA-pi21 and AA-4RQ are equivalent to genotypes H and A, respectively, in Fig. 1a.

Development of QTL pyramids. We first crossed the four NILs pairwise, and then selected progeny homozygous for resistance alleles at two QTLs by using the PCRbased markers indicated in Supp. Fig. S1. Next, we crossed plants homozygous for resistance alleles at two QTLs. In total, we developed eleven genotypes representing all possible combinations of two or more resistance QTL alleles. We then selected two to four lines per genotype. In a glasshouse inoculation test, three QTL pyramids-one with pi21 and $q B R 4-2$ (genotype code E in Fig. 1A), one with pi21, $q B R 4-2$, and Pi34 (code B), and one with all four QTL resistance alleles (code A; also called AA-4RQ) were used to test the effect of combining minor QTLs with the major QTL pi21.

Genotyping. Total DNA was extracted from leaf samples by using the CTAB method, and restriction fragment length polymorphism (RFLP) analysis was conducted as previously described ${ }^{9}$. Most of the simple sequence repeat (SSR) markers were obtained from previous report $\mathrm{s}^{30-32}$. The sequences of the other primer pairs (forward $5^{\prime}-3^{\prime} /$ reverse $\left.5^{\prime}-3^{\prime}\right)$ of PCR-based markers were as follows: ID04-25, GCCCAGGAGCAACAGAGT/TTCCGATGGTGATTTCATACT; ID04-26, TGGTTGGCGATTGACAGC/GCGGTCATGTAAATTGCCTAA; and ID01-47, TTTGCGGTTCGAGGATGTTT/GCCCATTGGTGGTTCCTCT. All markers were analysed in $2 \%$ to $3 \%$ agarose gel.

Evaluation of resistance to $M$. oryzae in the field. Resistance to $M$. oryzae was assessed during six growing seasons (2004-2008 and 2011) in an experimental field at the Aichi Agricultural Research Center, Mountainous Region Institute (MARI) (Inabu, Toyota, Aichi, Japan). The field had high levels of M. oryzae, and the predominant fungal race was 007.0. The lesion area (percentage of total leaf area) of 60- to 70-day-old plants was scored according to a reference scale. The score of each line was standardized as described previously ${ }^{13}$, and the arcsine-transformed value was used for statistical testing. Resistance was likewise tested in 2009 and 2011 in an experimental field at the National Institute of Agrobiological Sciences (Tsukuba, Ibaraki, Japan), where the predominant fungal race was 037.3.

To monitor $R$-gene-mediated resistance under field conditions, we tested eight NILs that each contain one of eight $R$-genes-Pik, Pik-m, Piz, Pizt, Pita, Pita2, Pib, or $P i i$-in the genetic background of the susceptible cultivar Sasanishiki ${ }^{33}$ at MARI along with Sasanishiki. Sasanishiki and the eight NILs all contain the R-gene Pia. Plants containing $R$-genes show either a resistant or a susceptible infection type when challenged with a given $M$. oryzae isolate, and the lesion area of resistant plants is nearly zero. However, most lines showed susceptible-type lesions in the test at MARI (Supp. Table S1). This suggests the presence of virulent pathogen races in this field, and we assumed that lesion area reflects the frequency of these virulent races. The inconsistent nature of $R$-gene-mediated resistance clearly demonstrates the potential risk of resistance breakdown when plants with a single $R$-gene are grown in the same area for several years ${ }^{3}$.

Glasshouse inoculation tests. We assessed resistance to nine $M$. oryzae isolates by using 35-day-old plants grown in a glasshouse maintained at $28 / 24^{\circ} \mathrm{C}$ (day/night) in three replications. Eight differential fungal isolates representing $M$. oryzae populations prevalent in Japan and a ninth isolate (IBOS8-1-1) that aggressively infects plants carrying Pi34 ${ }^{34}$ were used (Supp. Table S3). Fungal spores were suspended at a concentration of $1-2 \times 10^{6}$ spores $/ \mathrm{mL}$ in water containing $0.1 \%$ Tween 20, and then sprayed on plants at $0.6 \mathrm{~mL} /$ plant. The plants were then incubated in a humid chamber (relative humidity $>95 \%$ ) for $20 \mathrm{~h}$ at $24^{\circ} \mathrm{C}$ and transferred to the glasshouse until scoring. Images of infected leaves were obtained at 7 days after inoculation on a colour image scanner (Canon 4400F); images were scanned at $1200 \mathrm{dpi}$ and saved in RGB JPEG format. The images were transformed into the CIE-Lab colour space with software used in a previous study ${ }^{11}$. Each pixel was classified as either diseased (red) or undiseased (green) according to its a* value (one of the three components of the CIE-Lab colour space), and the percentage of lesion area was calculated as the ratio of the infected area to the total leaf area by using the previously described software ${ }^{11}$. The number of lesions per image was counted by eye on a computer display, and the number of lesions per 2.8 million pixels (the average size of a leaf at $1200 \mathrm{dpi}$ ) was used as the number of lesions per leaf.

To test the temperature dependence of the QTLs, we grew three sets of three genotypes (the recurrent parent and NILs for pi21 and qBR4-2) in the glasshouse under the conditions described above for 3 weeks. Each set was then transferred to one of three growth chambers $\left(20,24\right.$ or $\left.28^{\circ} \mathrm{C}\right)$ for 1 week before inoculation and then inoculated as described above.

To test the effect of ethylene and SA signalling on QTL-mediated resistance, we grew three sets of three genotypes (the recurrent parent and NILs for pi21 and Pi34) and inoculated them as described above except that they were soaked in water containing $300 \mu \mathrm{M} 2$-aminoisobutyric acid (AIB; an antagonist of ethylene biosynthesis), or $100 \mu \mathrm{M} \mathrm{SA}$, or $500 \mu \mathrm{M} \mathrm{SA}$, or water alone for $24 \mathrm{~h}$ until just before inoculation. For each genotype, the average lesion areas of treated plants were compared with that of the untreated (water only) control.

Fungal growth on leaf sheath epidermal tissue of 48-day-old plants was histologically characterized as previously described ${ }^{35}$. A previous study detected apparent differences between cells with and without pi21 at $96 \mathrm{~h}$ after inoculation, but not at $40 \mathrm{~h}^{35}$, and in a preliminary survey, we did not detect any differences among Aichiasahi, AA-pi21, and AA-4RQ at $33 \mathrm{~h}$. Thus, we examined cells at $45-48 \mathrm{~h}$ after 
inoculation. We evaluated $M$. oryzae biomass in leaves after inoculation by using realtime PCR as described previously ${ }^{36}$. We infected 28 -day-old plants with fungal races that were either compatible (003.0) or incompatible (001.0) with the Pia genes in the genotypes used. Fungal spores were prepared at a concentration of $8-11 \times$ $10^{4}$ spores $/ \mathrm{mL}$ in water containing $0.1 \%$ Tween 20 . We measured the amount of fungal DNA in DNA extracted from infected leaf tissue samples 4 days after inoculation by using the fungal repetitive element Pot2 (Z33638); the primers were SP6F (ACGACCCGTCTTTACTTATTTGG) and SP6R (AAGTACGCTTGGTTTTGTTGGAT $)^{37}$. DNA quantities were standardized against a rice ubiquitin gene $(\mathrm{AK} 121590)^{38}$. The experiment was performed twice.

Expression of pathogenesis-related genes. After the inoculation test, total RNA was isolated from leaves of 35-day-old plants by using an RNeasy Plant Mini Kit (Qiagen) and reverse-transcribed with primer dT18 and a First-Strand cDNA Synthesis Kit (GE Healthcare) according to the manufacturers' instructions. Quantitative real-time PCR was performed by using THUNDERBIRD SYBR qPCR Mix (Toyobo) and a 7900HT Fast Real-time PCR System (Applied Biosystems) according to the manufacturers' instructions. The genes and sequences of PCR primers (forward $5^{\prime}-3^{\prime} /$ reverse $5^{\prime}-3^{\prime}$ ) were Probenazole 1 (PBZ1; Os12t00555500), GTGGTTGTGTTTATGTGCCTTTCT/TGCCTCTCTTTATTCACCCATTG, and Pathogenesisrelated 2 (PR2; Os01t0940700), GCGCTTCAAGATGGCAAAG/GGCCGCAACTCCAAGGA. The expression level of riboubiquitin 2 (Os02t0161900), detected by primer pair GAGCCTCTGTTCGTCAAGTA/ACTCGATGGTCCATTAAACC, was used to standardize the RNA sample for each analysis. The RT-PCR and quantitative PCR assays were performed at least three times for each experimental line. In preliminary surveys, we did not detect any significant differences in the expression level of $P R$ genes among three genotypes (genotypes in Fig. 1a and Table 1: AA, Aichiasahi; H, AA-pi21; A, AA-4RQ) at 20-24 h after inoculation, so we focused on time points of 28,32 , and $36 \mathrm{~h}$.

Statistical tests. The individual effects of each allele and their significance levels were determined by ANOVA. A general linear model (GLM) was used to compare means among genotypes. All analyses were performed by using SPSS Statistics 19 (IBM).

1. Jones, J. D. \& Dangl, J. L. The plant immune system. Nature 444, 323-239 (2006).

2. Bonman, J. M., Khush, G. S. \& Nelson, R. J. Breeding rice for resistance to pests. Annu. Rev. Phytopathol. 30, 507-528 (1992).

3. Kiyosawa, S. Genetic and epidemiological modeling of breakdown of plant disease resistance. Annu. Rev. Phytopathol. 20, 93-117 (1982).

4. Poland, J. A., Balint-Kurti, P. J., Wisser, R. J., Pratt, R. C. \& Nelson, R. J. Shades of gray: the world of quantitative disease resistance. Trends Plant Sci. 14, 21-29 (2009).

5. Kou, Y. \& Wang, S. Broad-spectrum and durability: understanding of quantitative disease resistance. Curr. Opin. Plant Biol. 13, 181-185 (2010).

6. Saka, N. A rice (Oryza sativa L.) breeding for field resistance to blast disease (Pyricularia oryzae) in Mountainous Region Agricultural Research Institute, Aichi Agricultural Research Center of Japan. Plant Prod. Sci. 9, 3-9 (2006).

7. Miyamoto, M., Yano, M. \& Hirasawa, H. Mapping of quantitative trait loci conferring blast field resistance in the Japanese upland rice variety Kahei. Breed. Sci. 54, 257-261 (2001).

8. Wu, J. L. et al. Genetic control of rice blast resistance in the durably resistant cultivar Gumei 2 against multiple isolates. Theor. Appl. Genet. 111, 50-56 (2005).

9. Fukuoka, S. \& Okuno, K. QTL analysis and mapping of pi21, a recessive gene for field resistance to rice blast in Japanese upland rice. Theor. Appl. Genet. 103, 185-190 (2001).

10. Ballini, E. et al. A genome-wide meta-analysis of rice blast resistance genes and quantitative trait loci provides new insights into partial and complete resistance. MPMI 21, 859-868 (2008)

11. Fukuoka, S. et al. Loss of function of a proline-containing protein confers durable disease resistance in rice. Science 325, 998-1001 (2009).

12. Zenbayashi-Sawata, K. et al. Genetic and physical mapping of the partial resistance gene, Pi34, to blast in rice. Phytopathology 97, 598-602 (2007).

13. Fukuoka, S. et al. A multiple gene complex on rice chromosome 4 is involved in durable resistance to rice blast. Theor. Appl. Genet. 125, 551-599 (2012).

14. Wisser, R. J., Sun, Q., Hulbert, S. H., Kresovich, S. \& Nelson, R. J. Identification and characterization of regions of the rice genome associated with broadspectrum, quantitative disease resistance. Genetics 169, 2277-2793 (2005).

15. Xu, X. et al. Rice blast resistance gene Pikahei-1 $(\mathrm{t})$, a member of a resistance gene cluster on chromosome 4 , encodes a nucleotide-binding site and leucine-rich repeat protein. Mol. Breeding 34, 691-700 (2014).

16. van Loon, L. C., Geraats, B. P. J. \& Linthorst, H. J. M. Ethylene as a modulator of disease resistance in plants. Trends Plant Sci. 11, 184-191 (2006).

17. Vlot, A. C., Dempsey, D. A. \& Klessig, D. F. Salicylic Acid, a Multifaceted Hormone to Combat Disease. Annu. Rev. Phytopathol. 47, 177-206 (2009).

18. Silverman, P. et al. Salicylic Acid in Rice (Biosynthesis, Conjugation, and Possible Role). Plant Physiol. 108, 633-639 (1995).

19. Greenberg, J. T. \& Yao, N. The role and regulation of programmed cell death in plant-pathogen interactions. Cell. Microbiol. 6, 201-211 (2004).
20. Fukuoka, S. et al. Multiple functional polymorphisms in a single disease resistance gene in rice enhance durable resistance to blast. Sci Rep. 4, 4550 (2014).

21. Nguyen, T. T. et al. Pi35( $t$ ), a new gene conferring partial resistance to leaf blast in the rice cultivar Hokkai 188. Theor. Appl. Genet. 113, 697-704 (2006).

22. Fu, D. et al. A kinase-START gene confers temperature-dependent resistance to wheat stripe rust. Science 323, 1357-1360 (2009).

23. Zhu, Y., Qian, W. Q. \& Hua, J. Temperature Modulates Plant Defense Responses through NB-LRR Proteins. PLoS Pathog 6, e1000844 (2010).

24. Negeri, A. et al. Characterization of temperature and light effects on the defense response phenotypes associated with the maize Rp1-D21 autoactive resistance gene. BMC Plant Biol. 13, 106 (2013).

25. Ezuka, A. Field resistance of rice varieties to rice blast disease. Rev. Plant Prot. Res. 5, 1-21 (1972).

26. Kankanala, P., Czymmek, K. \& Valent, B. Roles for rice membrane dynamics and plasmodesmata during biotrophic invasion by the blast fungus. Plant Cell 19, 706-724 (2007)

27. Kiyosawa, S. [Disease resistance 2 - Blast disease] Collected data of plant genetic resources Vol. 1 [Matsuo, T. (ed.)] [365-368] (Kodansha Scientific, Tokyo, 1989).

28. Jannink, J. L., Lorenz, A. J. \& Iwata, H. Genomic selection in plant breeding: from theory to practice. Brief Funct. Genomics 9, 166-177 (2010).

29. Poland, J. A. \& Rife, T. W. Genotyping-by-sequencing for plant breeding and genetics. Plant Genome 5, 92-102 (2012).

30. Temnykh, S. et al. Computational and experimental analysis of microsatellites in rice (Oryza sativa $\mathrm{L}$.): frequency, length variation, transposon associations, and genetic marker potential. Genome Res. 11, 1441-1452 (2001)

31. McCouch, S. R. et al. Development and mapping of 2240 new SSR markers for rice (Oryza sativa L.). DNA Res. 9, 199-207 (2002).

32. IRGSP. The map-based sequence of the rice genome. Nature 436, 793-800 (2005).

33. Abe, S. Breeding of a blast resistant multiline variety of rice, Sasanishiki BL. JARQ 38, 149-154 (2004).

34. Zenbayashi-Sawata, K., Ashizawa, T. \& Koizumi, S. Pi34-AVRPi34: a new genefor-gene interaction for partial resistance in rice to blast caused by Magnaporthe grisea. J. Gen. Plant Pathol. 71, 395-401 (2005).

35. Koga, H., Dohi, K., Nakayachi, O. \& Mori, M. A novel inoculation method of Magnaporthe grisea for cytological observation of the infection process using intact leaf sheaths of rice plants. Physiol. Mol. Plant Pathol. 64, 67-72 (2004).

36. Hayashi, N. et al. Durable panicle blast-resistance gene $P b 1$ encodes an atypical CC-NBS-LRR protein and was generated by acquiring a promoter through local genome duplication. Plant J 64, 498-510 (2010).

37. Berruyer, R., Poussier, S., Kankanala, P., Mosquera, G. \& Valent, B. Quantitative and qualitative influence of inoculation methods on in planta growth of rice blast fungus. Phytopathology 96, 346-355 (2006).

38. Matsubara, K. et al. Ehd3, encoding a plant homeodomain finger-containing protein, is a critical promoter of rice flowering. Plant J. 66, 603-612 (2011).

\section{Acknowledgments}

We thank A. Takahashi, H. Inoue, E. Minami and T. Hayashi for helpful comments, and M. Matsuoka for critical reading of the manuscript. This work was supported by grants from the Ministry of Agriculture, Forestry and Fisheries of Japan (Development of Mitigation and Adaptation Techniques to Global Warming in the Sectors of Agriculture, Forestry, and Fisheries, Rice-2001; Genomics for Agricultural Innovation QT4004, GB1004, QTL2002, and RGB1101). We thank editors from ELSS, Inc. (http://elss.co.jp/en/) for editing the manuscript.

\section{Author contributions}

S.F. designed the experiments and wrote the manuscript. S.F. and K.E. developed the NILs and QTL-combination lines. S.F., N.H. and Y.Y. performed phenotyping in glasshouse inoculation tests. S.F., N.S., Y.M. and R.M. performed phenotyping in field tests. H.K. performed histological analysis. U.Y. performed expression analysis. M.Y. provided advice on the experiments and the manuscript.

\section{Additional information}

Supplementary information accompanies this paper at http://www.nature.com/ scientificreports

Competing financial interests: The authors declare no competing financial interests. How to cite this article: Fukuoka, S. et al. Gene pyramiding enhances durable blast disease resistance in rice. Sci. Rep. 5, 7773; DOI:10.1038/srep07773 (2015).

This work is licensed under a Creative Commons Attribution-NonCommercialShareAlike 4.0 International License. The images or other third party material in this article are included in the article's Creative Commons license, unless indicated otherwise in the credit line; if the material is not included under the Creative Commons license, users will need to obtain permission from the license holder in order to reproduce the material. To view a copy of this license, visit http:// creativecommons.org/licenses/by-nc-sa/4.0/ 